\title{
Stress scolaire, soutien social et burnout à l'adolescence : quelles relations?
}

\section{School stress, social support and burnout in adolescence: How are they connected?}

\section{Estrés escolar, apoyo social y agotamiento en la adolescencia, qué relación?}

\author{
Nicolas Meylan, Pierre-André Doudin, Denise Curchod-Ruedi, Jean-Philippe \\ Antonietti et Philippe Stephan
}

Volume 43, numéro 2, automne 2015

Le stress à l'école

URI : https://id.erudit.org/iderudit/1034489ar

DOI : https://doi.org/10.7202/1034489ar

Aller au sommaire du numéro

Éditeur(s)

Association canadienne d'éducation de langue française

ISSN

1916-8659 (numérique)

Découvrir la revue

Citer cet article

Meylan, N., Doudin, P.-A., Curchod-Ruedi, D., Antonietti, J.-P. \& Stephan, P. (2015). Stress scolaire, soutien social et burnout à l'adolescence : quelles relations? Éducation et francophonie, 43(2), 135-153.

https://doi.org/10.7202/1034489ar

\section{Résumé de l'article}

Récemment décrit comme un syndrome d'épuisement, de cynisme et d'inadéquation à l'école, le burnout scolaire suscite un intérêt grandissant parmi les professionnels de l'école. Ce phénomène a été associé à plusieurs variables comme le stress scolaire et le soutien social fourni par les parents et les enseignants. Cependant, nous en savons encore peu sur la façon dont ces variables interagissent. L'objectif de notre étude est d'explorer les effets modérateurs et médiateurs du stress scolaire et du soutien social afin de mieux comprendre leurs liens avec le burnout scolaire. Un questionnaire évaluant ces variables a été rempli par 441 adolescents de Suisse francophone. Tout d'abord, les analyses montrent que le soutien social ne modère pas l'effet du stress sur le burnout scolaire. Ensuite, il apparaît que le stress scolaire a un effet médiateur dans la relation entre le soutien social et le burnout scolaire. De plus, cet effet varie selon la source de soutien. D’une part, le stress médiatise partiellement la relation entre le soutien des parents et le burnout scolaire. D'autre part, le stress médiatise complètement le lien entre le soutien des enseignants et le burnout scolaire. Ainsi, il semblerait que les adolescents bénéficiant d'un bon niveau de soutien des parents et des enseignants perçoivent les exigences scolaires comme moins stressantes, ce qui diminue le risque de burnout.
Tous droits réservés () Association canadienne d'éducation de langue française, 2015
Cedocument est protége par la loi sur le droit d'auteur. L'utilisation des services d’Érudit (y compris la reproduction) est assujettie à sa politique d'utilisation que vous pouvez consulter en ligne. 


\section{Stress scolaire, soutien social et burnout à l'adolescence: quelles relations?}

\section{Nicolas MEYLAN}

Institut de psychologie, Université de Lausanne, Suisse

Unité d'enseignement et de recherche "Développement de l'enfant à l'adulte", Haute école pédagogique du canton de Vaud, Suisse

\section{Pierre-André DOUDIN}

Institut de psychologie, Université de Lausanne, Suisse

Unité d'enseignement et de recherche "Développement de l'enfant à l'adulte », Haute école pédagogique du canton de Vaud, Suisse

\section{Denise CURCHOD-RUEDI}

Unité d'enseignement et de recherche "Développement de l'enfant à l'adulte», Haute école pédagogique du canton de Vaud, Suisse

\section{Jean-Philippe ANTONIETTI}

Institut de psychologie, Université de Lausanne, Suisse

\section{Philippe STEPHAN}

Faculté de biologie et de médecine, Université de Lausanne, Suisse

\section{RÉSUMÉ}

Récemment décrit comme un syndrome d'épuisement, de cynisme et d'inadéquation à l'école, le burnout scolaire suscite un intérêt grandissant parmi les professionnels de l'école. Ce phénomène a été associé à plusieurs variables comme le stress 
scolaire et le soutien social fourni par les parents et les enseignants. Cependant, nous en savons encore peu sur la façon dont ces variables interagissent. L'objectif de notre étude est d'explorer les effets modérateurs et médiateurs du stress scolaire et du soutien social afin de mieux comprendre leurs liens avec le burnout scolaire. Un questionnaire évaluant ces variables a été rempli par 441 adolescents de Suisse francophone. Tout d'abord, les analyses montrent que le soutien social ne modère pas l'effet du stress sur le burnout scolaire. Ensuite, il apparait que le stress scolaire a un effet médiateur dans la relation entre le soutien social et le burnout scolaire. De plus, cet effet varie selon la source de soutien. D'une part, le stress médiatise partiellement la relation entre le soutien des parents et le burnout scolaire. D'autre part, le stress médiatise complètement le lien entre le soutien des enseignants et le burnout scolaire. Ainsi, il semblerait que les adolescents bénéficiant d'un bon niveau de soutien des parents et des enseignants perçoivent les exigences scolaires comme moins stressantes, ce qui diminue le risque de burnout.

\section{ABSTRACT}

\section{School stress, social support and burnout in adolescence: how are they connected?}

Nicolas MEYLAN

Institute of Psychology, University of Lausanne, Switzerland

"Child and Adult Development" Teaching and Research Unit, University of Teacher Education, Lausanne, Switzerland

Pierre-André DOUDIN

Institute of Psychology, University of Lausanne, Switzerland

"Child and Adult Development" Teaching and Research Unit, University of Teacher Education, Lausanne, Switzerland

Denise CURCHOD-RUEDI

"Child and Adult Development" Teaching and Research Unit, University of Teacher Education, Lausanne, Switzerland

Jean-Philippe ANTONIETTI

Institute of Psychology, University of Lausanne, Switzerland

Philippe STEPHAN

Faculty of Biology and Medicine, University of Lausanne, Switzerland

Recently described as a syndrome of exhaustion, cynicism and inadequacy, school burnout is arousing growing interest among school professionals. This phenomenon was associated with several variables, such as school stress and the social 
support provided by parents and teachers. However, we still know little about how these variables interact. The objective of this study is to explore the moderating and mediating effects of school stress and social support to better understand their connections with school burnout. A questionnaire evaluating these variables was completed by 441 adolescents from Francophone Switzerland. First, the analyses show that social support does not moderate the effect of stress on school burnout. Then it appears that school stress has a mediating effect on the relationship between social support and school burnout. This effect also varies according to the source of support. On one hand, stress partially mediates the relationship between parental support and school burnout. On the other hand, stress completely mediates the connection between teacher support and school burnout. Thus, it seems that adolescents who benefit from a good level of support from parents and teachers perceive school demands as less stressful, which lowers the risk of burnout.

\section{RESUMEN}

\section{Estrés escolar, apoyo social y agotamiento en la adolescencia, qué relación?}

Nicolas MEYLAN

Instituto de Sicología, Universidad de Lausana, Suiza

Unidad de Enseñanza y de Investigación "Desarrollo del niño al adulto», Escuela Superior de Pedagogía Vaud, Suiza

Pierre-André DOUDIN

Instituto de Sicología, Universidad de Lausana, Suiza

Unidad de Enseñanza y de Investigación «Desarrollo del niño al adulto», Escuela Superior de Pedagogía Vaud, Suiza

Denise CURCHOD-RUEDI

Unidad de Enseñanza y de Investigación "Desarrollo del niño al adulto», Escuela Superior de Pedagogía Vaud, Suiza

Jean-Philippe ANTONIETTI

Instituto de Sicología, Universidad de Lausana, Suiza

Philippe STEPHAN

Facultad de Biología et de Medicina, Universidad de Lausana, Suiza

Recientemente descrito como un síndrome de agotamiento, de cinismo y de inadecuación a la escuela, el burnout escolar suscita un interés creciente entre los profesionales de la escuela. Dicho fenómenos ha sido asociado a diversas variables como el estrés escolar y el apoyo social ofrecido por los padres y los maestros. Sin 
embargo muy poco sabemos sobre la manera en que dichas variables interactuar. El objetivo del presente estudio es explorar los efectos moderadores y mediadores del estrés escolar y del apoyo social con el fin de comprender más cabalmente sus relaciones con el burnout escolar. Un cuestionario para evaluar dichas variables fue aplicado a 441 adolescentes de la Suiza francófona. Por principio, los análisis muestran que el apoyo social no modera el efecto del estrés sobre el burnout escolar. Después, parece ser que el estrés escolar tiene un efecto mediador en la relación entre el apoyo social y el burnout escolar. Además dicho efecto varía según la fuente de apoyo. Por otra parte, el estrés mediatiza totalmente la relación entre el apoyo de los maestros y el burnout escolar. Así pues, parecería que los adolescentes que benefician de un buen nivel de apoyo de parte de la familia y de los maestros, perciben las exigencias escolares como menos estresantes, lo que disminuye el riesgo de burnout.

\section{Introduction}

Si les exigences scolaires favorisent le développement psychique, étant à la fois structurantes et valorisantes, elles mettent aussi certains adolescents en difficulté (Golse, 2005). Cela est d'autant plus vrai dans nos sociétés occidentales où l'accroissement des impératifs de réussite et de performance met à l'épreuve les ressources narcissiques de l'individu et entraine une forme de stress pour les adolescents (Jeammet, 2007; Stephan, 2011). Comme le montre une étude épidémiologique sur la santé et le bien-être chez les jeunes, environ $23 \%$ des adolescents français, $28 \%$ des adolescents suisses et belges, $50 \%$ des adolescents canadiens et jusqu'à $68 \%$ des adolescents turcs se disent stressés par leur travail scolaire (Currie et al., 2012). Afin d'aborder la problématique du stress scolaire et son possible impact pathologique, le concept de burnout a récemment été appliqué aux adolescents à l'école. L'utilisation de ce concept chez l'élève peut paraître surprenante mais, comme le soulignent certains auteurs (Schaufeli, Martinez, Pinto, Salanova et Bakker, 2002), l'activité principale des élèves s'apparente à un travail: se rendre à l'école, suivre des cours, rendre des travaux, préparer des examens et atteindre des objectifs en vue d'obtenir une certification. En d'autres termes, être élève peut être considéré comme un métier où il faut faire face à un certain nombre d'exigences (Perrenoud, 2004).

Le burnout scolaire suscite un intérêt grandissant parmi les professionnels de l'école et de la santé, d'autant plus qu'un haut niveau de burnout est associé à la symptomatologie anxio-dépressive (Salmela-Aro, 2011; Zakari, Walburg et Chabrol, 2011) et augmente significativement le risque de décrochage scolaire (Bask et Salmela-Aro, 2013). Il nous paraît donc essentiel d'identifier les facteurs de risque et de protection du burnout scolaire, mais aussi de décrire la façon dont ces facteurs interagissent. En ce sens, l'exploration d'effets modérateurs ou médiateurs devrait permettre de mieux comprendre comment certaines variables influencent le niveau 
de burnout. Pour rappel, un modérateur est une variable qualitative ou quantitative qui affecte la direction ou l'intensité entre la variable dépendante et la variable indépendante. Par contraste, un médiateur décrit plutôt un processus à travers lequel la variable indépendante est susceptible d'influencer la variable dépendante. À noter également que, dans ce processus, l'effet de la variable indépendante sur la variable dépendante peut être direct ou indirect, auquel cas il est courant de dire que la relation est médiatisée par une tierce variable. Pour plus de détails sur ces effets, nous renvoyons le lecteur à l'article de Rascle et Irachabal (2001).

\section{Un burnout lié au travail scolaire}

Défini comme une réponse à un stress scolaire chronique, le burnout scolaire apparaît chez des élèves initialement engagés dans leur scolarité, qui n'arrivent plus à faire face aux exigences de l'école (Salmela-Aro, Savolainen et Holopainen, 2009). Selon Salmela-Aro (2011), ce phénomène se caractérise par (1) un épuisement face aux demandes de l'école qui renvoie au sentiment d'être débordé par la pression liée à l'école, avec notamment une fatigue chronique due aux inquiétudes et aux ruminations concernant le travail scolaire; (2) du cynisme envers l'école, qui renvoie à une attitude détachée ou indifférente face à l'école en général, avec une perte d'intérêt pour le travail scolaire et une incapacité à lui donner du sens; (3) un sentiment d'inadéquation en tant qu'élève, qui renvoie au sentiment de «ne pas être à la hauteur» dans son travail scolaire, un manque d'accomplissement dans le travail scolaire et à l'école en général.

De récents travaux indiquent un taux de prévalence allant de $6,7 \%$ à $15 \%$ pour le burnout scolaire sévère et permettent d'identifier certains déterminants de ce trouble (Meylan, Doudin, Curchod-Ruedi, Antonietti et Stephan, 2012; Salmela-Aro et Tynkkynen, 2012; Slivar, 2001). Il apparaît notamment que les filles sont les plus à risque de burnout, de même que les adolescents scolarisés dans des filières d'études à niveau d'exigence scolaire élevé (Salmela-Aro, Kiuru et Nurmi, 2008a; Meylan, Doudin, Curchod-Ruedi et Stephan, 2011). Enfin, par extension des travaux ayant montré, d'une part, que le stress lié au travail est un des principaux prédicteurs du burnout professionnel (Maslach, Schaufeli et Leiter, 2001) et, d'autre part, que le soutien social constitue un important facteur de protection contre ce trouble (Doudin, Curchod-Ruedi et Moreau, 2011), un intérêt particulier a été porté sur le stress lié à l'école et sur le soutien social dans la problématique du burnout scolaire.

\section{Stress scolaire et burnout}

Le stress scolaire peut être défini comme un état chronique chez des élèves qui se sont fixé des objectifs irréalistes ou dont la perception et les attentes de l'entourage dépassent leurs capacités (Ang et Huan, 2006).

Dans la continuité des travaux ayant associé le stress professionnel au burnout chez l'adulte (voir Maslach et al., 2001), plusieurs auteurs se sont intéressés aux associations entre le stress scolaire et le burnout chez l'adolescent. Sur le plan théorique, Salmela-Aro et al. (2009) ont proposé d'aborder le burnout scolaire comme un phénomène continu allant du stress au burnout sévère, suggérant ainsi le lien entre ces 
deux concepts. Sur le plan empirique, Zakari et al. (2011) mettent en évidence le fait que le stress scolaire est un prédicteur significatif et positif du burnout scolaire, en soulignant notamment qu'un haut niveau de stress lié à l'école est associé à un haut niveau de burnout scolaire. Dans une autre étude, Meylan et al. (2011) précisent ces associations en définissant plusieurs types de stress scolaire et leurs effets respectifs sur le burnout scolaire. Il apparaît notamment que le stress lié à la réussite et à l'avenir scolaire est le principal prédicteur de l'épuisement et du sentiment d'inadéquation, alors que le cynisme est plus influencé par le stress lié à la quantité de travail scolaire. Il semblerait donc que le stress scolaire constitue un important facteur de risque du burnout chez les élèves.

\section{Soutien social et burnout}

Demaray et Malecki (2002) définissent le soutien social comme la perception qu'a un individu des comportements généraux ou spécifiques de soutien adoptés par les personnes de son entourage et qui peuvent améliorer son fonctionnement ou le prémunir contre des effets indésirables.

Chez l'adulte, de nombreux travaux ont établi que le niveau et la satisfaction du soutien social sont négativement associés au burnout professionnel (Sundin, Hochwälder, Bildt et Lisspers, 2007; Doudin et al., 2011) et que ces associations varient selon la source de soutien (Halbesleben, 2006). Cependant, seules quelques études ont souligné les liens entre le soutien social et le burnout scolaire chez l'adolescent. Par exemple, Salmela-Aro, Kiuru, Pietikäinen et Jokela (2008b) montrent qu'un haut niveau de soutien de l'école est associé à un faible niveau de burnout scolaire. Dans une autre étude, Meylan, Doudin, Curchod-Ruedi et Stephan (2014) mettent en évidence le fait qu'un haut niveau de soutien des enseignants et des parents est négativement associé au burnout scolaire, et en particulier à la dimension du cynisme. Enfin, un bas niveau de soutien des parents et des enseignants est associé à davantage d'attitudes négatives et cyniques envers l'école (Rueger, Malecki et Demaray, 2010; Malecki et Bjorkman, 2007). Ces différents travaux soulignent le rôle potentiellement protecteur du soutien social contre le burnout scolaire. De plus, ils mettent en évidence l'importance de considérer l'effet spécifique de différentes sources de soutien comme les parents et les enseignants dans les études explorant les associations entre le soutien social et le burnout scolaire.

\section{Stress, soutien social et burnout}

D'après les travaux sur le burnout professionnel, il est largement admis que le stress associé au travail, le soutien social et le burnout sont liés (Maslach et al., 2001; Doudin et al. 2011; Halbesleben, 2006; Schaufeli, Leiter et Maslach, 2009). Cependant, le débat reste ouvert quant à la façon dont ces variables interagissent. Selon le modèle demandes-ressources (Bakker et Demerouti, 2007), le burnout apparaîtrait lorsque les ressources dont dispose un individu ne lui permettent plus de faire face aux exigences liées au travail et que ce déséquilibre persiste sur une longue période. Dans ce contexte, un haut niveau de soutien social permet de diminuer le stress lié aux exigences professionnelles et réduit le risque de burnout. Ainsi, comme 
le soulignent certains auteurs, le soutien social serait un modérateur de la relation entre le stress et le burnout (Maslach et al., 2001; Truchot, 2004). À l'inverse et selon le modèle de conservation des ressources (Hobfoll et Freedy, 1993; Halbesleben, 2006), le burnout serait la conséquence d'un stress chronique causé par un appauvrissement des ressources mises à la disposition d'un individu. Ainsi, un haut niveau de soutien social amène à percevoir les demandes professionnelles de façon moins contraignante et stressante, ce qui diminue le risque de burnout. En d'autres termes, le stress serait un médiateur de la relation entre le soutien social et le burnout (Cooper, Dewe et O’Driscoll, 2001; Kelley, 1994).

Chez l'adolescent, nous en savons encore peu sur les associations entre le stress lié à l'école, le burnout scolaire et le soutien social. Salmela-Aro et Upadyaya (2013) apportent un élément de réponse en montrant que le modèle Demandes-Ressources peut être appliqué aux élèves en contexte scolaire. Plus particulièrement, ces auteurs (Salmela-Aro et Upadyaya, 2013) suggèrent que le burnout scolaire serait la conséquence d'un processus d'appauvrissement de l'énergie de l'élève où des demandes scolaires trop importantes débordent les ressources dont dispose ce dernier. À l'inverse, soulignons que des auteurs s'intéressant à la pratique du sport à l'adolescence ont montré que le soutien social influence de façon indirecte la sévérité du burnout sportif à travers son impact sur le niveau de stress perçu (Raedeke et Smith, 2004).

La compréhension des processus pouvant mener au burnout ne se fait donc pas uniquement en identifiant les facteurs de risque ou de protection, mais également en explorant leurs interactions. Comme le soulignent Meylan et ses collaborateurs (2014), des études sont nécessaires afin d'examiner les effets modérateurs et médiateurs entre les différents facteurs associés au burnout scolaire.

\section{Problématique et hypothèses}

À notre connaissance, aucune étude n'a encore examiné les relations entre le soutien social, le stress lié à l'école et le burnout scolaire. Pourtant, dans la continuité des travaux sur le burnout professionnel chez l'adulte et le burnout sportif chez l'adolescent, nous pouvons supposer l'existence d'interactions entre ces variables.

Tout d'abord, en référence au modèle Demandes-Ressources du burnout, nous pouvons supposer que le soutien social modère ou médiatise la relation entre le stress et le burnout chez l'adolescent. Ensuite, par analogie aux travaux ayant montré que le stress médiatise la relation entre le soutien social et le burnout sportif à l'adolescence (Raedeke et Smith, 2004; Kelley, 1994), nous pouvons supposer que le stress scolaire médiatise la relation entre le soutien social et le burnout lié au travail scolaire. Enfin, le soutien des parents et le soutien des enseignants ayant des effets spécifiques sur le burnout scolaire (Meylan et al., 2014; Salmela-Aro et al., 2008), nous pouvons aussi supposer que les interactions entre le stress scolaire et le soutien social varient selon la source de soutien considérée. Nous faisons donc l'hypothèse (H1) que le soutien social modère l'effet du stress sur le burnout scolaire, (H2) que le soutien social médiatise la relation entre le stress et le burnout scolaire, (H3) que le stress scolaire médiatise la relation entre le soutien social et le burnout scolaire et (H4) que les effets modérateurs ou médiateurs varient selon la source du soutien 
social. Précisons encore que, pour chacune de ces hypothèses, nous contrôlons l'effet du genre sur le burnout scolaire. Et cela, car de précédents travaux ont mis en évidence le fait que les filles sont plus à risque de burnout scolaire que les garçons (Salmela-Aro et al., 2008a; Zakari, Walburg et Chabrol, 2011).

\section{Méthode}

\section{Population et procédure}

Notre échantillon se compose de 441 adolescents (223 filles et 218 garçons) recrutés dans plusieurs écoles publiques secondaires de Suisse francophone. Ces adolescents ont entre 13 et 18 ans (moyenne $=14,78$; écart type $=1,13$ ) et en sont aux dernières années de la scolarité obligatoire. Ils ont répondu collectivement et durant une période de classe à un questionnaire sur le stress lié à l'école, le soutien social et le burnout scolaire. Des consignes relatives à la passation ont été données oralement et nous avons aussi rappelé aux adolescents qu'ils pouvaient refuser de prendre part à l'exercice ou de répondre à certaines questions. Dans ces conditions, aucun jeune n'a refusé de participer, mais certains questionnaires nous ont été rendus avec des réponses en partie manquantes. Enfin, l'anonymat des élèves a été garanti et notre recherche respecte le code déontologique et éthique de la Société suisse de psychologie (SSP), notamment en ce qui concerne les autorisations d'accès aux données et l'accord des participants ainsi que de leurs représentants légaux.

\section{Mesures}

\section{Burnout scolaire}

Nous avons mesuré le burnout scolaire à l'aide du School Burnout Inventory (SBI - Salmela-Aro et al., 2009) dont la version française a récemment été validée (Meylan, Doudin, Curchod-Ruedi, Antonietti et Stephan, 2012). Ce questionnaire est composé de 9 items relatifs aux trois dimensions théoriques du burnout scolaire. L'épuisement face aux demandes de l'école est évalué par 4 items (p. ex.: «Je me sens débordé par mon travail scolaire»); le cynisme à l'égard du sens de l'école est mesuré par 3 items (p. ex. : «Je me demande continuellement si mon travail scolaire a du sens») et le sentiment d'inadéquation en tant qu'élève est évalué par 2 items (p. ex.: "J'ai souvent l'impression d'être insuffisant dans mon travail scolaire»). Les items sont cotés sur une échelle de Likert en 6 points, allant de $1=$ Complétement faux à $6=$ Complètement vrai, et permettent d'évaluer le burnout scolaire de façon globale et dimensionnelle. Les qualités psychométriques de la version française du SBI sont dans l'ensemble satisfaisantes, avec notamment une structure factorielle identique à celle de la version originale. Dans le cadre de cette étude, nous nous intéressons au burnout scolaire dans une perspective globale; c'est pourquoi nous utilisons uniquement le score total de burnout. Le coefficient de Cronbach $(\alpha=0,82)$ indique une cohérence interne satisfaisante. 


\section{Stress scolaire}

Nous avons évalué le stress scolaire à l'aide d'une version française du Adolescent Hassles Inventory (Bobo, Gilchrist, Elmer, Snow, et Schinke, 1986; validation française par Plancherel, Bettschart, Bolognini, Dumont et Halfon, 1997). Ce questionnaire composé de 59 items permet de mesurer la fréquence et l'intensité de tracas quotidiens relatifs à la vie familiale, la perception de soi, les relations avec les pairs et l'école. Les adolescents doivent indiquer s'ils ont vécu ou non ces différents tracas et, si oui, dans quelle mesure ils en ont été affectés au cours des six derniers mois. Les réponses sont données sur une échelle de Likert en 4 points, allant de $1=$ Pas du tout stressé à 4 = Très stressé. Dans le cadre de cette étude, nous utilisons uniquement 19 items en lien avec la scolarité. Comme l'ont souligné Meylan et al. (2011), ces items permettent d'évaluer le stress scolaire de façon globale, mais aussi dimensionnelle, en distinguant entre le stress lié à la réussite et à l'avenir scolaire (p. ex. être inquiet au sujet de mon avenir scolaire), le stress lié à la charge de travail scolaire (p. ex. avoir trop de travaux écrits à préparer pour l'école) et le stress lié aux relations à l'école (p. ex. être victime de racket à l'école). Dans le cadre de cette étude, nous abordons le stress de façon globale, raison pour laquelle nous utilisons uniquement le score total de stress scolaire. Le coefficient de Cronbach $(\alpha=0,84)$ indique une cohérence interne satisfaisante.

\section{Soutien social}

Nous avons évalué le soutien social à l'aide d'une version française du Child and Adolescent Social Support Scale (CASSS - Malecki et Demaray, 2002; adaptation française par Meylan et al., 2014). Ce questionnaire de 60 items permet de mesurer la perception qu'ont les adolescents du soutien social que leur fournissent les parents, les enseignants, les camarades de classe, le meilleur ami / la meilleure amie et l'école. À chaque source de soutien correspond une échelle de 12 items évaluant quatre types de soutien, à savoir émotionnel (3 items; p. ex. : "Mes parents m'écoutent lorsque j'ai besoin de parler»), informationnel (3 items; p. ex.: "Mes parents me donnent de bons conseils»), évaluatif (3 items; p. ex.: "Mes parents me font gentiment remarquer mes erreurs") et instrumental (3 items; p. ex. : "Mes parents prennent le temps de m'aider à faire des choix»). Les adolescents répondent aux différents items sur deux aspects: (1) la fréquence, évaluée à l'aide d'une échelle de Likert en 6 points allant de 1 = Jamais à 6 = Toujours; (2) l'importance, évaluée sur une échelle de Likert en 3 points allant de 1 = Peu important à 3 = Très important. À partir de ces évaluations, il est possible de calculer un score total de fréquence et d'importance du soutien par source (parent, enseignant, camarade de classe, meilleur(e) ami(e) et école), mais aussi des scores de fréquence et d'importance du soutien par type (émotionnel, informationnel, évaluatif et instrumental) pour une même source. Les qualités psychométriques de la version française du CASSS sont très satisfaisantes avec une structure factorielle identique à celle de la version originale et une bonne fiabilité des échelles. Dans le cadre de cette étude, nous ne tenons compte que des scores de fréquence étant donné que les scores d'importance sont davantage destinés à l'interprétation clinique (Malecki et Demaray, 2002). De plus, nous utilisons uniquement 
les items relatifs au soutien des parents et des enseignants, car ce sont les seules sources dont le score total de soutien perçu permet de prédire le niveau de burnout scolaire (Meylan et al., 2014). Les coefficients de Cronbach indiquent une cohérence interne satisfaisante pour le soutien des enseignants $(\alpha=0,89)$ et le soutien des parents $(\alpha=0,94)$.

\section{Analyses statistiques}

Les données manquantes ont été traitées par la méthode d'imputation HotDeck. Cette méthode, largement utilisée (pour une revue, voir Andridge et Little, 2010), permet de remplacer une valeur manquante par une valeur réelle issue d'un profil similaire dans la base de données.

L'effet modérateur du soutien social dans la relation entre le stress et le burnout scolaire a été examiné à l'aide d'une régression hiérarchique (voir Devereux, Hastings, Noone, Firth \&et Totsika, 2009). Puis, les effets médiateurs du stress et du soutien social ont été testés en suivant la procédure d'analyse two-step proposée par Anderson et Gerbing (1988). La première étape vise à examiner la validité du modèle de mesure à l'aide d'une analyse confirmatoire. La seconde étape consiste à évaluer le modèle structurel afin d'identifier les relations significatives entre le stress lié à l'école, le soutien social et le burnout scolaire. La qualité d'ajustement de ces modèles a été évaluée à l'aide des indices suivants (voir Hu et Bentler, 1999) : la statistique du $X^{2}$; un $X^{2}$ normé (CMIN/DF) inférieur ou égal à 3; un Root Mean Square Error of Approximation (RMSEA) et un Standarized Root Mean Square Residual (SRMR) inférieur ou égal à 0,08; un Comparative Fit Index (CFI) et un Tucker-Lewis Index (TLI) supérieur ou égal à 0,90; et le Akaike Information Criterion (AIC) dont la valeur la plus faible indique quel modèle s'ajuste le mieux aux données.

Afin de contrôler l'amplification des erreurs de mesure dues à la multiplication d'items par facteurs latents, nous avons, pour chaque questionnaire, regroupé les items relatifs à une même dimension. Toutes les analyses ont été réalisées à l'aide des logiciels R-statistics et AMOS 21 (Arbuckle, 2012).

\section{Résultats}

\section{Effets modérateurs du soutien social}

La régression hiérarchique permettant de tester l'effet modérateur du soutien social (H1) comprend deux blocs dans lesquels nous avons inséré les variables selon la méthode d'entrée forcée. Dans le bloc 1, nous avons introduit le genre afin de contrôler l'effet de cette variable sur le burnout scolaire. Dans le bloc 2, nous avons regroupé le stress scolaire, le soutien des parents et le soutien des enseignants. Dans le bloc 3, nous avons inséré les interactions stress X soutien des parents et stress X soutien des enseignants. Tous les scores ont été centrés réduits pour éviter les problèmes de colinéarité. Les résultats montrent que seul le bloc 2 augmente significativement la part de variance expliquée du burnout scolaire $\left(\Delta \mathrm{R}^{2}=0,35, p<0,001\right)$. L'absence d'effet significatif en ce qui concerne les interactions du bloc 3 suggère 
également que le soutien social n'est pas un modérateur de la relation entre le stress et le burnout scolaire.

\section{Effet médiateur du stress et du soutien social Modèle de mesure}

Le modèle de mesure comprend quatre facteurs latents (correspondant au score total de burnout, de stress, de soutien des parents et de soutien des enseignants) et 14 variables observées (qui renvoient aux sous-dimensions des échelles utilisées). Les indices obtenus pour ce modèle témoignent d'un ajustement très satisfaisant, $\mathrm{X}^{2}(71, \mathrm{~N}=441)=221,98, p<0,001 ; \mathrm{CMIN} / \mathrm{DF}=3,12 ; \mathrm{CFI}=0,95 ; \mathrm{TLI}=0,93 ; \mathrm{RMSEA}=$ 0,$07 ;$ SRMS $=0,05 ;$ AIC $=317,98$. Tous les coefficients de régression standardisés des indicateurs d'un même facteur latent sont significatifs ( $p s<0,001$ ), ce qui indique que les facteurs latents sont bien représentés par leurs indicateurs respectifs. En effet, (1) le burnout scolaire renvoie bien à l'épuisement, au cynisme et au sentiment d'inadéquation, (2) le stress scolaire est bien lié au succès et à l'avenir, à la surcharge de travail ainsi qu'aux relations à l'école, (3) le soutien des parents et celui des enseignants se caractérisent bien par du soutien de type émotionnel, informationnel, évaluatif et instrumental. De plus, comme nous pouvons le voir au tableau 1, tous les facteurs latents du modèle de mesure sont significativement corrélés.

Tableau 1. Intercorrélations entre les facteurs latents du modèle de mesure

\begin{tabular}{lcccc}
\hline Variables & $\mathbf{1}$ & $\mathbf{2}$ & $\mathbf{3}$ & $\mathbf{4}$ \\
\hline 1. Stress scolaire & $(0,84)$ & & & \\
\hline 2. Burnout scolaire & $0,56^{* *}$ & $(0,82)$ & & \\
\hline 3. Soutien des parents & $-0,21^{* *}$ & $-0,28^{* *}$ & $(0,94)$ & \\
\hline 4. Soutien des enseignants & $-0,23^{* *}$ & $-0,21^{* *}$ & $0,27^{* *}$ & $(0,89)$ \\
\hline
\end{tabular}

$\mathrm{N}=441$

Note: Tous les coefficients de corrélations sont significatifs au seuil de $p<0,01$. Les coefficients alpha de Cronbach sont indiqués entre parenthèses.

\section{Modèle structurel}

Nous avons vérifié nos hypothèses $\mathrm{H} 2$, $\mathrm{H} 3$ et $\mathrm{H} 4$ en testant quatre modèles alternatifs. Les indices d'ajustement de ces modèles sont rapportés dans le tableau 2. Le modèle M1, avec deux médiateurs et un chemin direct allant du stress au burnout scolaire, montre un bon ajustement aux données. Cependant, les coefficients standardisés des chemins allant du soutien social (des parents et des enseignants) au burnout scolaire sont non significatifs. De plus, les indices de modification indiquent une relation significative entre le soutien des enseignants et celui des parents. Nous avons donc testé un modèle sans médiation du soutien social (M2) en contraignant les chemins non significatifs de M1 à zéro et en ajoutant une corrélation entre le soutien des enseignants et celui des parents. Les indices d'ajustement de ce modèle sont 
satisfaisants et la valeur du AIC indique que le modèle M2 s'ajuste mieux aux données que le modèle M1.

Nous avons ensuite testé un autre modèle de médiation partielle (M3) avec le stress comme médiateur de la relation entre le soutien des enseignants et celui des parents et le burnout scolaire. Les indices de ce modèle sont relativement satisfaisants, mais le coefficient standardisé du soutien des enseignants sur le burnout scolaire n'est pas significatif. C'est pourquoi nous avons testé un modèle où, respectivement, le soutien des parents et des enseignants médiatise partiellement et totalement la relation entre le stress et le burnout scolaire (M4). Pour ce faire, nous avons contraint à zéro le chemin entre le soutien des enseignants et le burnout scolaire. L'ajustement de ce modèle est très satisfaisant avec une valeur AIC inférieure à celle des autres modèles. Nous avons donc retenu le modèle M4 comme étant le meilleur.

Figure 1. Modèle structural final

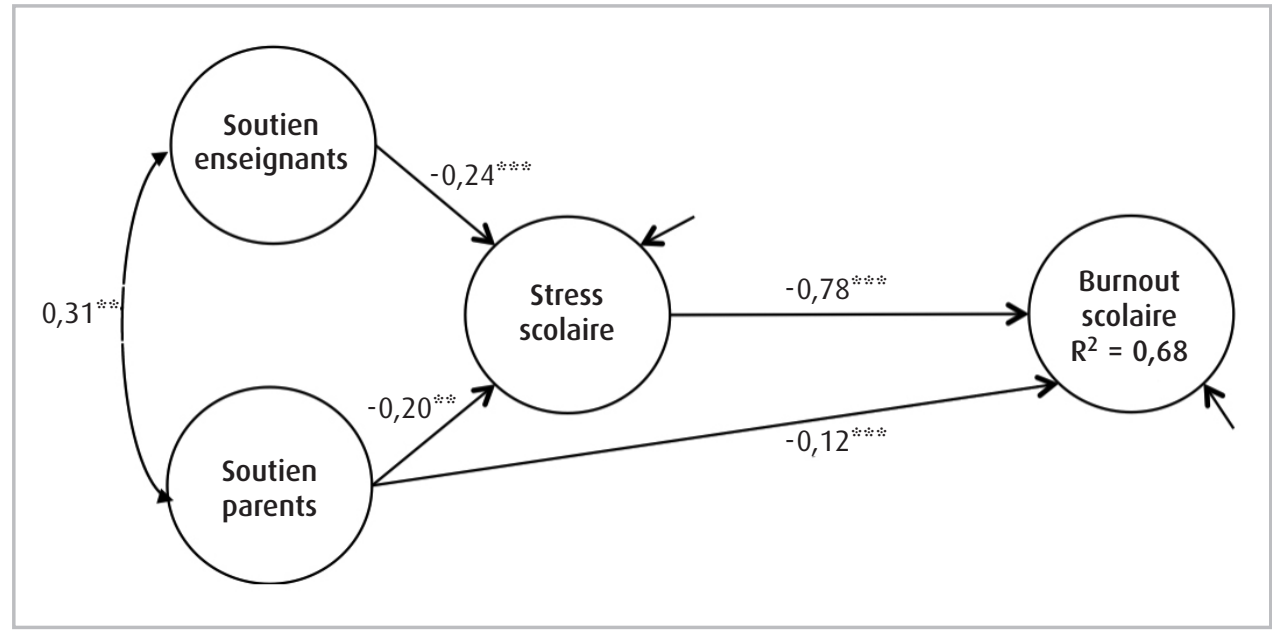

$\mathrm{p}<<0,05, * \mathrm{p}<0,01, * \mathrm{p}<0,001$

Note: Modèle M4 où le stress scolaire est un médiateur de la relation entre le soutien social et le burnout scolaire.

Le modèle final (M4) est présenté à la figure qui précède. Tout d'abord, il apparaît que le stress scolaire est un prédicteur direct et positif du burnout scolaire $(\Omega=$ $0,78, p<0,001)$. Ensuite, nous pouvons observer que le soutien des parents a un effet direct négatif sur le burnout $(\Omega=-0,12, p<0,05)$ et le stress scolaire $(\Omega=-0,20$, $p<0,01$ ), ce qui suggère que le stress scolaire médiatise partiellement la relation entre le soutien des parents et le burnout. Cette médiation a été vérifiée à l'aide de la procédure de rééchantillonnage (bootstrap) et il en résulte que le soutien des parents a un effet indirect sur le burnout en agissant sur le stress scolaire $(ß=-0,16,95 \%$ CI $[-0,27,-0,04])$. Enfin, il apparaît que le soutien des enseignants a un effet direct négatif sur le stress scolaire $(\Omega=-0,24, p<0,001)$, mais aucun effet sur le burnout, ce qui suggère que le stress scolaire médiatise complètement la relation entre le soutien des 
enseignants et le burnout. La vérification de cette médiation montre que le soutien des enseignants a un effet indirect sur le burnout par son action sur le stress scolaire $(ß=-19,95 \%$ CI $[-0,28,-0,08])$. Soulignons encore que nous avons procédé à une analyse multigroupe afin de vérifier si le modèle final a la même structure en fonction du genre. Il apparaît que le modèle sans contrainte et le modèle contraint ne diffèrent pas significativement $\left(\Delta \mathrm{X}^{2}=22,05, \mathrm{dl}=14, p>0,05\right)$, ce qui indique que le modèle final est valide pour les filles et pour les garçons.

\section{Tableau 2. Indices d'ajustement des modèles alternatifs}

\begin{tabular}{|c|c|c|c|c|c|c|c|c|}
\hline Modèle & $\mathbf{X}^{2}$ & df & CMIN/DF & CFI & TLI & RMSEA & SRMS & AIC \\
\hline M1 & 240,96 & 72 & 3,34 & 0,94 & 0,92 & 0,07 & 0,07 & 334,97 \\
\hline M2 & $226,92 \cdots$ & 73 & 3,11 & 0,95 & 0,93 & 0,07 & 0,05 & 318,92 \\
\hline M3 & 221,98 & 71 & 3,13 & 0,95 & 0,93 & 0,07 & 0,05 & 317,89 \\
\hline M4 & $222,11^{*}$ & 72 & 3,08 & 0,95 & 0,93 & 0,07 & 0,05 & 316,12 \\
\hline
\end{tabular}

.... $p<0,001$

\section{Discussion}

Il est largement admis que le burnout est un trouble lié au stress (Maslach et al., 2001). Cependant, l'un des défis consiste à comprendre pourquoi certains adolescents sont plus stressés par les exigences scolaires et peuvent montrer des signes de burnout scolaire, récemment décrit comme un syndrome d'épuisement, de cynisme et d'inadéquation par rapport à l'école. L'objectif de notre étude était d'explorer les associations entre le soutien social, le stress et le burnout scolaire.

Tout d'abord, nous nous sommes intéressés à l'effet modérateur du soutien social. Nos résultats montrent qu'il n'existe aucune interaction entre le soutien des parents ou des enseignants et le stress scolaire, ce qui contredit notre première hypothèse selon laquelle le soutien social modère l'effet du stress scolaire sur le burnout (H1). Toutefois, cela va dans le sens des travaux qui ont montré que le soutien social ne modère pas systématiquement la relation entre stresseurs et troubles psychopathologiques à l'adolescence (pour une revue, voir Grant, Compas, Thurm, McMahon, Gipson et al., 2006). Par conséquent, si le soutien social des parents et des enseignants constitue un facteur de protection contre le burnout scolaire (Meylan et al., 2014), il semblerait que ce ne soit pas dû à un effet modérateur de la relation entre le stress scolaire et le burnout.

Ensuite, nous nous sommes penchés sur les effets de médiations entre le stress et le soutien social. Les résultats de l'examen des modèles alternatifs ne nous permettent pas de confirmer l'hypothèse d'un effet médiateur du soutien social dans la relation entre le stress scolaire et le burnout $(\mathrm{H} 2)$. Il semblerait donc que le processus 
par lequel le stress influence le burnout scolaire ne puisse pas être expliqué par la perception que les adolescents ont du soutien des parents et des enseignants, ce qui va dans le sens des travaux ayant montré que le soutien social ne médiatise pas l'effet du stress sur la santé mentale des adolescents (Yarcheski et Mahon, 1999). À l'inverse, les relations mises en évidence dans le modèle structurel final confirment notre hypothèse selon laquelle le stress scolaire médiatise la relation entre le soutien social et le burnout scolaire (H3). Il semblerait donc que le soutien social permette de réduire le risque de burnout lié à l'école de façon directe mais aussi indirecte en diminuant le niveau de stress scolaire. Enfin, nous avons observé que l'effet médiateur du stress est partiel lorsque le soutien vient des parents, alors qu'il est total lorsque le soutien vient des enseignants. Cela confirme notre hypothèse selon laquelle les effets médiateurs varient en fonction de la source de soutien (H4). Il apparaît donc que le burnout scolaire est influencé de façon directe par le soutien des parents, ce qui va dans le sens de précédents travaux (Salmela-Aro, 2011; Meylan et al., 2014). Nous mettons également en évidence le fait que le burnout scolaire est influencé de façon indirecte par le soutien des parents et des enseignants et que ces effets s'observent aussi bien chez les filles que chez les garçons. En d'autres termes, les adolescents et les adolescentes qui se sentent soutenus par leurs parents et leurs enseignants percevraient les exigences scolaires de façon peu stressante, ce qui diminue leur risque de burnout scolaire.

Dans l'ensemble, ces résultats soulignent que le stress lié à l'école a un effet médiateur dans la relation entre le soutien social et le burnout scolaire à l'adolescence. De plus, ils montrent que cet effet médiateur est différent selon que l'on considère le soutien des parents ou des enseignants. Nous rejoignons ici, d'une part, les travaux ayant souligné que le stress médiatise la relation entre le soutien social et le burnout lié au sport chez les adolescents et les adultes (Kelley, 1994; Raedeke et Smith, 2004) et, d'autre part, les auteurs postulant que le burnout apparaîtrait à la suite d'un stress causé par un appauvrissement des ressources à disposition (Hobfoll et Freedy, 1993; Halbesleben, 2006).

Cette étude comporte plusieurs limites. Tout d'abord, il convient d'être prudent en ce qui concerne la portée heuristique et la généralisation des résultats, étant donné que la technique d'échantillonnage «tout-venant» n'assure pas la représentativité de notre échantillon. Ensuite, il est important de souligner que la causalité ne peut pas être testée dans cette étude en raison de la nature transversale des données. Des études longitudinales devraient favoriser une meilleure compréhension des liens causaux entre le soutien social, le stress et le burnout scolaire. Enfin, l'utilisation d'autoquestionnaires implique certains biais méthodologiques (voir Razavi, 2001) et ne permet qu'une évaluation subjective du soutien social, du stress et du niveau de burnout scolaire. Bien que l'évaluation subjective de ces variables soit essentielle par nature (Rueger et al., 2010; Plancherel et al., 1997; Salmela-Aro et al., 2009), des études ultérieures pourraient bénéficier de l'utilisation de mesures subjectives et objectives, notamment en ce qui concerne le stress scolaire. 


\section{Conclusion}

Cette recherche met en évidence les associations entre le soutien social, le stress et le burnout scolaire et permet d'étendre notre compréhension de ce syndrome à l'adolescence. Nous montrons que le stress scolaire est un médiateur de la relation entre le soutien social et le burnout scolaire et que ce médiateur agit différemment selon la source de soutien. Plus particulièrement, nous soulignons le fait que les parents et les enseignants sont des sources dont le soutien est, respectivement, partiellement et totalement médiatisé par le stress scolaire. En d'autres termes, le niveau de soutien des parents influence la sévérité du burnout scolaire de façon directe et indirecte, alors que le niveau de soutien des enseignants influence ce syndrome uniquement de façon indirecte par son effet sur le niveau de stress scolaire.

Ces résultats ont des implications en termes de recherche et de prévention. Tout d'abord, cette étude est la première à montrer un effet de médiation du stress scolaire dans la problématique du burnout à l'adolescence. D'un point de vue théorique, cela permet d'envisager le burnout scolaire selon le modèle du stress et du burnout proposé par Kelley (1994) ainsi que selon le modèle de la conservation des ressources de Hobfoll et Freedy (1993) dont la pertinence a été soulignée par les travaux sur le burnout lié à l'activité sportive et professionnelle. Dans une perspective de prévention du burnout scolaire, nous pensons qu'il faudrait renforcer le soutien des parents et des enseignants dans la mesure où un haut niveau de soutien social réduit directement et indirectement le risque de burnout scolaire. Ce travail pourrait se faire notamment durant la formation des enseignants en soulignant davantage l'importance des attitudes des enseignants dans le développement scolaire et psychologique des adolescents. Nous pensons aussi qu'il ne s'agit pas uniquement d'augmenter le soutien à disposition, mais aussi d'attirer davantage l'attention des adolescents sur le soutien qui leur est offert et notamment le soutien qu'ils peuvent recevoir de la part de leurs enseignants. Ce travail pourrait par exemple être fait au sein de l'école dans le cadre d'une prévention primaire du burnout scolaire. Si de nombreuses pistes de réflexion restent à explorer, cette étude apporte un éclairage utile pour la mise en place de mesures préventives contre le burnout scolaire chez les adolescents.

\section{Références bibliographiques}

ANDERSON, J. C. et GERBING, D. W. (1988). Structural equation modeling in practice: A review and recommended two-step approach. Psychological Bulletin, 103(3), 411-423. doi: 10.1037/0033-2909.103.3.411

ANDRIDGE, R. R. et LITTLE, R. J. (2010). A review of hot deck imputation for survey non-response. International Statistical Review, 78(1), 40-64. doi:

$$
\text { 10.1111/j.1751-5823.2010.00103.x }
$$


ANG, R. P. et HUAN, V. S. (2006). Relationship between academic stress and suicidal ideation: Testing for depression as a mediator using multiple regression. Child Psychiatry and Human Development, 37(2), 133-143. doi: 10.1007/s10578-0060023-8

ARBUCKLE, J. L. (2012). Amos 21. User's guide. Chicago, IL: IBM SPSS.

BAKKER, A. et DEMEROUTI, E. (2007). The Job Demands-Resources model: State of the art. Journal of Managerial Psychology, 22(3), 309-328. doi: $10.1108 / 02683940710733115$

BASK, M. et SALMELA-ARO, K. (2013). Burned out to drop out: Exploring the relationship between school burnout and school dropout. European Journal of Psychology of Education, 28(2), 511-528. doi : 10.1007/s10212-012-0126-5

BOBO, J. K., GILCHRIST, L. D., ELMER, J. F., SNOW, W. H. et SCHINKE, S. P. (1986). Hassles, role strain, and peer relations in young adolescents. Journal of Early Adolescence, 6(4), 339-352. doi: 10.1177/0272431600020001005

BOLLEN, K. A. (1989). Structural equations with latent variables. New York: Wiley and Sons.

COOPER, C. L., DEWE, P. J. et O’DRISCOLL, M. P. (2001). Organizational stress: A review and critique of theory, research, and applications. Newbury Park, NJ : Sage Publications.

CURRIE, C., ZANOTTI, C., MORGAN, A., CURRIE, D., DE LOOZE, M., ROBERTS, C. et al. (dir.) (2012). Social determinants of health and well-being among young people. Health Behaviour in School-aged Children (HBSC) study: International report from the 2009/2010 survey (Health Policy for Children and Adolescents, No. 6). Copenhague, Danemark: WHO Regional Office for Europe.

DEMARAY, M. K. et MALECKI, C. K. (2002). The relationship between perceived social support and maladjustment for students at risk. Psychology in the Schools, 39(3), 305-316. doi: 10.1002/pits.10018

DOUDIN, P.-A., CURCHOD-RUEDI, D. et MOREAU, J. (2011). Le soutien social comme facteur de protection de l'épuisement des enseignants. Dans P.-A. Doudin, D. Curchod-Ruedi, L. Lafortune et N. Lafranchise (dir.), La santé psychosociale des enseignants et des enseignantes (p. 11-37). Québec, Canada: Presses de l'Université du Québec.

DEVEREUX, J. M., HASTINGS, R. P., NOONE, S. J., FIRTH, A. et TOTSIKA, V. (2009). Social support and coping as mediators or moderators of the impact of work stressors on burnout in intellectual disability support staff. Research in Developmental Disabilities, 30(2), 367-377. doi: 10.1016/j.ridd.2008.07.002

GOLSE, B. (2005). L'impact des exigences scolaires au fil des âges. Nervure, 1, 1-2. 
GRANT, K. E., COMPAS, B. E., THURM, A. E., MCMAHON, S. D., GIPSON, P. Y., CAMPBELL, A. J. et al. (2006). Stressors and child and adolescent psychopathology: Evidence of moderating and mediating effects. Clinical Psychology Review, 26(3), 257-283. doi: 10.1016/j.cpr.2005.06.011

HALBESLEBEN, J. B. (2006). Sources of social support and burnout: A meta-analytic test of the conservation of resources model. The Journal of Applied Psychology, 91(5), 1134-1145.

HOBFOLL, S. E. et FREEDY, J. (1993). Conservation of resources: A general stress theory applied to burnout. Dans W. B. Schaufeli, C. Maslach et T. Marek (dir.), Professional burnout: Recent developments in theory and research (p. 115-133). Washington, DC: Taylor \& Francis.

HU, L. et BENTLER, P. M. (1999). Cutoff criteria for fit indexes in covariance structure analysis: conventional criteria versus new alternatives. Structural Equation Modeling, 6(1), 1-55. doi: 10.1080/10705519909540118

JEAMMET, P. (2007). Le moi à l'épreuve de la liberté. Neuropsychiatrie de l'enfance et de l'adolescence, 55(1), 321-328. doi: 10.1016/j.neurenf.2007.07.010

KELLEY, B. C. (1994). A model of stress and burnout in collegiate coaches: Effects of gender and time of season. Research Quarterly for Exercise and Sport, 65(1), 48-58.

MALECKI, C. K. et BJORKMAN, S. M. (2007). Relationships among academic stress, social support, and internalizing and externalizing behavior in adolescence. Illinois: Northern Illinois University.

MALECKI, C. K. et DEMARAY, M. K. (2002). Measuring perceived social support: Development of the child and adolescent social support scale. Psychology in the Schools, 39, 1-18. doi: 10.1002/pits.10004

MASLACH, C., SCHAUFELI, W. B. et LEITER, M. P. (2001). Job burnout. Annual Review of Psychology, 52, 397-422. doi: 10.1111/1467-8721.01258

MEYLAN, N., DOUDIN, P.-A., CURCHOD, D. et STEPHAN, P. (2011). School burnout in adolescents: Differences in background variables and exploration of schoolrelated stress at the end of compulsory schooling. Ricerche di Psicologia, 4, 539-563. doi: 10.3280/RIP2011-004006

MEYLAN, N., DOUDIN, P.-A., CURCHOD, D. et STEPHAN, P. (2014). Burnout scolaire et soutien social : l'importance du soutien des parents et des enseignants. Psychologie française. doi: 10.1016/j.psfr.2014.01.003

MEYLAN, N., DOUDIN, P.-A., CURCHOD-RUEDI, D., ANTONIETTI, J.-P. et STEPHAN, P. (2012, novembre). School Burnout Inventory: structure factorielle, fiabilité et validité de la version française. Poster présenté à la Journée de la recherche de la Faculté des sciences sociales et politiques, Université de Lausanne. 
PERRENOUD, P. (2004). Métier d'élève et sens du travail scolaire. Issy-lesMoulineaux, France: ESF.

PLANCHEREL, B., BETTSCHART, W., BOLOGNINI, M., DUMONT, M. et HALFON, O. (1997). Influence comparée des événements existentiels et des tracas quotidiens sur la santé psychique à la préadolescente. Neuropsychiatrie de l'enfance et de l'adolescence, 45(3), 26-38.

RASCLE, N. et IRACHABAL, S. (2001). Médiateurs et modérateurs: implications théoriques et méthodologiques dans le domaine du stress et de la psychologie de la santé. Le travail humain, 64(2), 97-118.

RAEDEKE, T. D. et SMITH, A. L. (2004). Coping resources and athlete burnout: An examination of stress mediated and moderation hypotheses. Journal of Sport and Exercise Psychology, 26(4), 525-541.

RAZAVI, T. (2001). Self-report measures: An overview of concerns and limitations of questionnaire use in occupational stress research. Southampton, R.-U.: University of Southampton.

RUEGER, S. Y., MALECKI, C. K. et DEMARAY, M. K. (2010). Relationship between multiple sources of perceived social support and psychological and academic adjustment in early adolescence: Comparisons across gender. Journal of Youth and Adolescence, 39, 47-61. doi: 10.1007/s10964-008-9368-6

SALMELA-ARO, K. (2011). Le burnout des élèves. Dans D. Curchod-Ruedi, P.-A. Doudin, L. Lafortune et N. Lafranchise (dir.), La santé psychosociale des élèves (p. 33-45). Québec, Canada: Presses de l'Université du Québec.

SALMELA-ARO, K. et TYNKKYNEN, L. (2012). Gendered pathways in school burnout among adolescents. Journal of Adolescence, 35(4), 929-939. doi: 10.1016/j.adolescence.2012.01.001

SALMELA-ARO, K. et UPADYAYA, K. (2013). School burnout and engagement in the context of demands-resources model. British Journal of Educational Psychology. doi: 10.1111/bjep.12018

SALMELA-ARO, K., KIURU, N. et NURMI, J.-E. (2008a). The role of educational track in adolescents' school burnout: A longitudinal study. British Journal of Educational Psychology, 78(4), 663-689. doi: 10.1348/000709908X281628

SALMELA-ARO, K., KIURU, N., LESKINEN, E. et NURMI, J.-E. (2009). School burnout inventory: Reliability and validity. European Journal of Psychological Assessment, 25(1), 48-57. doi: 10.1027/1015-5759.25.1.48

SALMELA-ARO, K., KIURU, N., PIETIKÄINEN, M. et JOKELA, J. (2008b). Does school matter? The role of school context for school burnout. European Psychologist, 13, 1-13. doi: 10.1027/1016-9040.13.1.12 
SALMELA-ARO, K., SAVOLAINEN, H. et HOLOPAINEN, L. (2009). Depressive symptoms and school burnout during adolescence: Evidence from two cross-lagged longitudinal studies. Journal of Youth and Adolescence, 38(10), 1316-1327. doi: 10.1007/s10964-008-9334-3

SCHAUFELI, W. B., LEITER, M. P. et MASLACH, C. (2009). Burnout: 35 years of research and practice. Career Development International, 14(3), 204-220. doi: $10.1108 / 13620430910966406$

SCHAUFELI, W. B., MARTINEZ, I., PINTO, A. M., SALANOVA, M. et BAKKER, A. (2002). Burnout and engagement in university students: A cross-national study. Journal of Cross-Cultural Psychology, 33(5), 464-481. doi: $10.1177 / 0022022102033005003$

SLIVAR, B. (2001). The syndrome of burnout, self-image, and anxiety with grammar school students. Horizons of Psychology, 10(2), 21-32.

STEPHAN, P. (2011). La prévention des dépendances à l'école. Dans D. CurchodRuedi, P.-A. Doudin, L. Lafortune et N. Lafranchise (dir.), La santé psychosociale des élèves (p. 149-162). Québec, Canada: Presses de l'Université du Québec.

SUNDIN, L., HOCHWÄLDER, J., BILDT, C. et LISSPERS, J. (2007). The relationship between different work-related sources of social support and burnout among registered and assistant nurses in Sweden: A questionnaire survey. International Journal of Nursing Studies, 44(5), 758-769. doi: 10.1016/j.ijnurstu.2006.01.004

TRUCHOT, D. (2004). Épuisement professionnel et burnout: concepts, modèles, interventions. Paris, France: Dunod.

YARCHESKI, A. et MAHON, N. E. (1999). The moderator-mediator role of social support in early adolescents. Western Journal of Nursing Research, 21(5), 685-698.

ZAKARI, S., WALBURG, V. et CHABROL, H. (2011). Stress et sentiment d'épuisement scolaire chez des lycéens français. Dans D. Curchod-Ruedi, P.-A. Doudin, L. Lafortune et N. Lafranchise (dir.), La santé psychosociale des élèves (p. 47-64). Québec, Canada: Presses de l'Université du Québec. 